\title{
A missense variant in EZH2 is associated with colorectal cancer risk in a Chinese population
}

\author{
Huihui Li ${ }^{1,2}$, Chunxiao Chang ${ }^{2}$, Yuhong Shang ${ }^{3}$, Ling Qiang², Baoxuan Zhang ${ }^{2}$, Bing \\ $\mathrm{Bu}^{2}$, Guohua Ren ${ }^{2}$, Lihua Song ${ }^{2}$, Mao Shang ${ }^{4}$ and Jinming $\mathbf{Y u}^{4}$ \\ ${ }^{1}$ Shandong University, Jinan, Shandong Province, China \\ ${ }^{2}$ Department of Medical Oncology, Shandong Cancer Hospital Affiliated to Shandong University, Shandong Academy of \\ Medical Sciences, Jinan, Shandong Province, China \\ ${ }^{3}$ Clinical Laboratory, Shandong Cancer Hospital Affiliated to Shandong University, Shandong Academy of Medical Sciences, \\ Jinan, Shandong Province, China \\ ${ }^{4}$ Department of Radiation Oncology, Shandong Cancer Hospital Affiliated to Shandong University, Shandong Academy of \\ Medical Sciences, Jinan, Shandong Province, China
}

Correspondence to: Jinming Yu, email: sdyujinming@163.com

Keywords: colorectal cancer, susceptibility, enhancer of zeste 2 polycomb repressive complex 2 subunit, exon region, missense variant

Received: August 28, $2017 \quad$ Accepted: September 23, $2017 \quad$ Published: October 16, 2017

Copyright: Li et al. This is an open-access article distributed under the terms of the Creative Commons Attribution License 3.0 (CC BY 3.0), which permits unrestricted use, distribution, and reproduction in any medium, provided the original author and source are credited.

\section{ABSTRACT}

Colorectal cancer (CRC) ranks the fifth leading cause of cancer death in China. EZH2 is a member of Polycomb-group (PCG) family and associated with transcriptional repression and cancer development. In this study, we report the association between a missense variant in EZH2 and risk of CRC. Through a systematic selection of variants in EZH2, we identified rs2302427 in the exon region of EZH2 and genotyped this variant in 852 CRC patients and 1,303 healthy controls using Taqman genotyping assay. The association between this variant and CRC risk was calculated using logistic regression with adjustment of sex, age, smoking status and drinking status. The result showed that rs2302427 was significantly associated with CRC susceptibility under an additive model $(P=0.0068)$. Compared with CC genotype carriers, CG genotype and GG genotype carriers were associated with risk of CRC with odds ratio being 0.78 (95\% CI: 0.63-0.96, $P=0.0198$ ) and 0.54 (95\% CI: 0.24-1.18, $P=0.1224$ ), respectively. When stratified by sex, age, smoking status or drinking status, significant associations were observed only in younger individuals ( $O R=0.67,95 \% \mathrm{CI}: 0.50-0.89, P=0.0067)$ or smokers ( $O R=0.65,95 \% \mathrm{CI}: 0.48-0.88, P=0.0051)$. This study provides new insights into the personalized prevention of colorectal cancer.

\section{INTRODUCTION}

Colorectal cancer (CRC) is one of the most common malignancies worldwide and ranks the third leading cause of cancer in both men and women [1-3]. Although environmental factors such as over-nutrition, obesity and smoking have been associated with the development of sporadic CRC $[4,5]$, only a small portion of exposed individuals develop CRC, indicating that an individual's genetic makeup also play an important role in the etiology of CRC [6,7]. Previous studies have identified many genetic polymorphisms that were correlated with colorectal cancer risk in the Chinese population using both genomewide association $[8,9]$ and candidate strategy $[10,11]$.

Enhancer of zeste 2 polycomb repressive complex 2 subunit (EZH2), a histone methyl transferase subunit of polycomb repressor complex 2 (PRC2), a complex that methylates lysine 27 of histone $\mathrm{H} 3$ (H3K27) to promote transcriptional silencing $[12,13]$. EZH2 is recurrently mutated in many types of cancer and play an important role in cancer proliferation and progression [14-16]. 
Polymorphisms in EZH2 have been identified to be associated with multiple cancers, such as bladder cancer, Oral squamous cell carcinoma and colorectal cancer [1720]. However, most of these studies only focus on variants in the promoter region of $\mathrm{EZH} 2$, the association between missense variants in EZH2 and risk of colorectal cancer were not interrogated.

Here we report a case control study for a missense variant in EZH2 and colorectal cancer susceptibility. This study consists of $852 \mathrm{CRC}$ patients and 1,303 controls and the result showed that rs2302427 was significantly associated with colorectal cancer risk.

\section{RESULTS}

\section{Select characteristic of study subjects}

The distributions of select characteristics including sex, age, smoking status and drinking status of the CRC patients and controls participated in this study were shown in Table 1. 852 CRC patients and 1,303 healthy controls were used in the analysis. There were $67.6 \%$ and $65.2 \%$ males in cases and controls, respectively. The average age of cases and controls were 60.7 and 63.5, respectively. There are $40.8 \%$ smokers and $30.2 \%$ drinkers among cases while there are $36.5 \%$ smokers and $29.2 \%$ drinkers among controls.

\section{Association between rs2302427 and risk of CRC}

We found only one variant located in the exon region of $\mathrm{EZH} 2$ with minor allele frequency $>1 \%$ in Chinese population. There are five variants that are in high LD $\left(r^{2}>0.6\right)$ with rs2302427. All these SNPs are in the intron region of EZH2 with no functional clue. The genotype frequencies of this variant and its association with risk of CRC were shown in Table 2. The rs2302427 was significantly associated with CRC susceptibility with odds ratio being 0.77 in an additive model (95\% CI: 0.640.93, $P=0.0068)$. Compared with $\mathrm{CC}$ genotype carriers, CG genotype and GG genotype carriers were associated with risk of $\mathrm{CRC}$ with odds ratio being 0.78 (95\% CI: $0.63-0.96, P=0.0198)$ and 0.54 (95\% CI: 0.24-1.18, $P=0.1224)$, respectively.

\section{Stratified analysis of rs2302427 and risk of CRC}

We performed stratified analyses by sex, age, smoking status and drinking status to evaluate the effects of variant genotypes on the risk of CRC (Table 3). Among the younger individuals ( $\leq 60$ years), rs2302427 was significantly associated with CRC susceptibility with an odds ratio of 0.67 (95\% CI: $0.50-0.89, P=0.0067)$. However, the association were not significant for older individuals $(>60$ years $)(\mathrm{OR}=0.85,95 \% \mathrm{CI}$ : 0.66-1.10, $P=0.2175)$. When stratified by smoking status, rs 2302427 was significantly associated with CRC risk in smokers, but not in nonsmokers, with the OR being 0.65 (95\% CI: 0.48 $0.88, P=0.0051)$ and 0.87 (95\% CI: $0.68-1.11, P=0.2508)$, respectively.

\section{Functional prediction of rs2302427}

The rs2302427 variant located in the 185th amino acid of EZH2 with an amino acid change of Asp to His. The PolyPhen-2 (http://genetics.bwh.harvard.edu/pph2/) predicts that this variant have a $60.3 \%$ possible to influence EZH2 based on physical and comparative considerations. This variant was also predicted to have function with a AA change score being 85 in MutationTaster2 (score ranging from 0-215).

\section{DISCUSSION}

This is the first study of genetic polymorphism in exon region of EZH2 and risk of colorectal cancer. Through a case control study consist of 852 cases and 1,303 controls of Chinese population, we identified that rs2302427 (Asp185His) was significantly associated with risk of colorectal cancer. We found that the frequency of GC and CC genotypes of rs2302427 were significantly lower in CRC patients than the healthy controls. Moreover, through a stratification analysis, the significant association was found only in younger patients and smokers, but not in older patients or nonsmokers. These results indicated an important role of EZH2 in CRC carcinogenesis.

EZH2 encodes a member of the Polycomb-group $(\mathrm{PcG})$ family, function as a subunit of polycomb repressor complex 2 (PRC2), which involved in maintaining the transcriptional repressive state of genes over successive cell generations. PRC2 catalyses the mono-, di- and trimethylation of lysine 27 of histone H3 (H3K27me1, $\mathrm{H} 3 \mathrm{~K} 27 \mathrm{me} 2$ and $\mathrm{H} 3 \mathrm{~K} 27 \mathrm{me} 3$, respectively) [12]. $\mathrm{H} 3 \mathrm{~K} 27 \mathrm{me} 3$ is a hallmark of transcriptional silencing and is thought to result in gene repression together with PRC1, which play important roles in cancer $[21,22]$. EZH2 was initially shown to have oncogenic functions; however, many studies in recent years has shown that PRC2 also has tumor suppressive effects in cancer [23]. EZH2 has also been considered as a biomarker for multiple cancers [24]. Polymorphisms in EZH2 has also been reported to be associated with multiple cancer, including colorectal cancer [17-20]. In this study, we found a novel missense variant (rs2302427) that was correlated with decreased risk of colorectal cancer. A previous study has proved that rs2302427 variant decreased histone methyltransferase activity of EZH2, making this association biological possible $[25,26]$.

Our study also has some limitations. First, the study has constrained by a relatively small sample size that we did not investigate rare missense variants in EZH2. Second, the present case-control study only tested in one 
Table 1: Select characteristics of subjects participated in this study

\begin{tabular}{lcc}
\hline & Cases $(\mathbf{n}=\mathbf{8 5 2})$ & Controls $(\mathbf{n}=\mathbf{1 , 3 0 3})$ \\
\hline $\begin{array}{l}\text { Age (years), mean } \pm \text { S.D. } \\
\text { Gender, n (\%) }\end{array}$ & $60.7 \pm 13.1$ & $63.5 \pm 9.6$ \\
$\quad$ Male & $576(67.6)$ & $850(65.2)$ \\
$\quad$ Female & $276(32.4)$ & $453(34.8)$ \\
Smoking status & & $827(63.5)$ \\
$\quad$ Nonsmoker & $504(59.2)$ & $476(36.5)$ \\
$\quad$ Smoker & $348(40.8)$ & \\
Drinking status & & $923(70.8)$ \\
$\quad$ Nondrinker & $595(69.8)$ & $380(29.2)$ \\
$\quad$ Drinker & $257(30.2)$ & \\
\hline
\end{tabular}

Table 2: Association between rs2302427 and risk of CRC in a Chinese population

\begin{tabular}{|c|c|c|c|c|c|c|c|c|}
\hline SNP & Chr & Position & Location & Genotype & $\begin{array}{c}\text { Cases No. } \\
(\%)\end{array}$ & Controls No. (\%) & OR $(95 \% \text { CI })^{a}$ & $P^{\mathbf{a}}$ \\
\hline \multirow[t]{5}{*}{ rs2302427 } & 7 & 148525904 & Asp $>$ His & $\mathrm{CC}$ & $670(78.6)$ & $961(73.8)$ & $\begin{array}{c}1.00 \\
\text { (Reference) }\end{array}$ & \\
\hline & & & & $\mathrm{CG}$ & $173(20.3)$ & $319(24.5)$ & $\begin{array}{c}0.78(0.63- \\
0.96)\end{array}$ & 0.0198 \\
\hline & & & & GG & $9(1.1)$ & $23(1.8)$ & $\begin{array}{c}0.54(0.24- \\
1.18)\end{array}$ & 0.1224 \\
\hline & & & & $\mathrm{CG}+\mathrm{GG}$ & $182(21.4)$ & $342(26.2)$ & $\begin{array}{c}0.76(0.62- \\
0.94)\end{array}$ & 0.0096 \\
\hline & & & & $\begin{array}{l}\text { Additive } \\
\text { model }\end{array}$ & & & $\begin{array}{c}0.77(0.64- \\
0.93)\end{array}$ & 0.0068 \\
\hline
\end{tabular}

${ }^{a}$ Calculated by logistic regression with adjustment for sex, age, smoking status and drinking status.

Table 3: Result of stratification analysis for $\mathbf{r s 2 3 0 2 4 2 7}$

\begin{tabular}{llc}
\hline & OR (95\% CI) & $P$ \\
\hline Male & $0.83(0.66-1.04)$ & 0.1130 \\
Female & $0.63(0.44-0.90)$ & 0.0102 \\
$>60$ years & $0.85(0.66-1.10)$ & 0.2175 \\
$\leq 60$ years & $0.67(0.50-0.89)$ & 0.0067 \\
Smoker & $0.65(0.48-0.88)$ & 0.0051 \\
Nonsmoker & $0.87(0.68-1.11)$ & 0.2508 \\
Drinker & $0.78(0.54-1.12)$ & 0.1720 \\
Nondrinker & $0.76(0.60-0.95)$ & 0.0149 \\
\hline
\end{tabular}


center. More replication studies should be performed to confirm the association between rs2302427 and risk of CRC in the future. Third, although previous studies have proved that rs2302427 may influence EZH2, more functional experiments should be performed to investigate how this variant affect CRC susceptibility. Third, the conclusion that the variant was only associated with risk of colorectal cancer in younger patients and smokers need to be confirmed in larger samples.

In summary, through screen single nucleotide polymorphisms (SNPs) in the exon region of EZH2 gene, we found that rs2302427 is associated with susceptibility to CRC. Compared to rs2302427C allele, individuals with rs2302427G was associated with lower CRC risk, especially in younger patients and smokers. This study provided new evidence for the precise medicine of CRC.

\section{MATERIALS AND METHODS}

\section{Study subjects}

CRC patients were enrolled from Shandong Cancer Hospital Affiliated to Shandong University, Jinan, China between January 1st, 2014 to August 30th, 2016. Controls samples were selected from a community cancer screening program for early detection conducted in the same region during the same period as cases were collected. All CRC patients were confirmed primary $\mathrm{CRC}$, without any radiotherapy or chemotherapy treatment prior to blood samples collected. Both cases and controls were Han Chinese descent. The informed consent was obtained from every participant at recruitment and peripheral blood samples and demographic characteristics such as gender, age and ethnicity were collected by interviewers. This study was conducted under the approval of the institutional review boards of Shandong Cancer Hospital Affiliated to Shandong University.

\section{SNP selection and genotyping}

We searched for variants in the exon region of EZH2 with minor allele frequency $(\mathrm{MAF})>0.01$ in Chinese Han Beijing (CHB) population. One variant (rs2302427, TCATCATCGT [C/G] ATCATCATTA in forward strand) was selected and was genotyped in a Chinses population consist of 852 cases and 1,303 controls. Genomic DNA were extracted from at least $1 \mathrm{ml}$ peripheral blood sample collected from each participant at recruitment. Genotyping were performed using Taqman SNP Genotyping Assay (Applied Biosystems). The case and control samples were mixed in the plates, and persons who performed the genotyping assay were not aware of case or control status.

\section{Statistical analysis}

Unconditional multivariate logistic regression analysis with adjustment of sex, age, smoking status and drinking status was performed to assess the association between rs2302427 and risk of CRC. All statistical analyses were performed using R software. All tests were two-sided and $P<0.05$ were considered significant.

\section{Abbreviations}

Colorectal cancer, CRC; Enhancer of zeste 2 polycomb repressive complex 2 subunit, EZH2; Polycomb-group, PcG; Polycomb repressor complex 2, PRC2; Lysine 27 of histone H3, H3K27; Monomethylation of lysine 27 of histone H3, H3K27me1; Dimethylation of lysine 27 of histone H3, H3K27me2; Trimethylation of lysine 27 of histone H3, H3K27me3; Single nucleotide polymorphisms, SNPs; Chinese Han Beijing, CHB

\section{Author contributions}

Huihui Li performed experiments, analyzed data and wrote the paper; Chunxiao Chang, Yuhong Shang, Ling Qiang and Baoxuan Zhang collected the samples and performed experiments; Bing Bu, Guohua Ren, Lihua Song and Mao Shang collected the information of the cases and controls and analyzed data, and Jinming Yu designed experiments, analyzed data and wrote the paper.

\section{ACKNOWLEDGMENTS}

The authors would like to thank China Postdoctoral Science Foundation for the financial grant.

\section{CONFLICTS OF INTEREST}

No potential conflicts of interest were disclosed by the authors.

\section{FUNDING}

This work was supported by the 58th China Postdoctoral Science Foundation (Grant No.: 2015M582100).

\section{REFERENCES}

1. Siegel RL, Miller KD, Jemal A. Cancer statistics, 2017. CA: A Cancer Journal for Clinicians. 2017; 67: 7-30. https://doi. org/10.3322/caac.21387.

2. Siegel RL, Miller KD, Fedewa SA, Ahnen DJ, Meester RGS, Barzi A, Jemal A. Colorectal cancer statistics, 2017. CA Cancer J Clin. 2017; 67: 177-93. https://doi. org/10.3322/caac.21395.

3. Kleer CG, Cao Q, Varambally S, Shen R, Ota I, Tomlins SA, Ghosh D, Sewalt RG, Otte AP, Hayes DF, Sabel MS, Livant D, Weiss SJ, et al. EZH2 is a marker of aggressive breast cancer and promotes neoplastic transformation of 
breast epithelial cells. Proc Natl Acad Sci U S A. 2003; 100: 11606-11. https://doi.org/10.1073/pnas.1933744100.

4. Song M, Garrett WS, Chan AT. Nutrients, foods, and colorectal cancer prevention. Gastroenterology. 2015; 148: 1244-60 e16. https://doi.org/10.1053/j.gastro.2014.12.035.

5. Bailie L, Loughrey MB, Coleman HG. Lifestyle Risk Factors for Serrated Colorectal Polyps: A Systematic Review and Meta-analysis. Gastroenterology. 2017; 152: 92-104. https://doi.org/10.1053/j.gastro.2016.09.003.

6. Lichtenstein P, Holm NV, Verkasalo PK, Iliadou A, Kaprio J, Koskenvuo M, Pukkala E, Skytthe A, Hemminki K. Environmental and heritable factors in the causation of cancer--analyses of cohorts of twins from Sweden, Denmark, and Finland. N Engl J Med. 2000; 343: 78-85. https://doi.org/10.1056/NEJM200007133430201.

7. de la Chapelle A. Genetic predisposition to colorectal cancer. Nat Rev Cancer. 2004; 4: 769-80. https://doi. org/10.1038/nrc1453.

8. Jia WH, Zhang B, Matsuo K, Shin A, Xiang YB, Jee SH, Kim DH, Ren Z, Cai Q, Long J, Shi J, Wen W, Yang G, et al. Genome-wide association analyses in East Asians identify new susceptibility loci for colorectal cancer. Nat Genet. 2013; 45: 191-6. https://doi.org/10.1038/ng.2505.

9. Zeng C, Matsuda K, Jia WH, Chang J, Kweon SS, Xiang YB, Shin A, Jee SH, Kim DH, Zhang B, Cai Q, Guo X, Long J, et al. Identification of Susceptibility Loci and Genes for Colorectal Cancer Risk. Gastroenterology. 2016; 150: 1633-45. https://doi.org/10.1053/j.gastro.2016.02.076.

10. Lou J, Gong J, Ke J, Tian J, Zhang Y, Li J, Yang Y, Zhu Y, Gong Y, Li L, Chang J, Zhong R, Miao X. A functional polymorphism located at transcription factor binding sites, rs6695837 near LAMC1 gene, confers risk of colorectal cancer in Chinese populations. Carcinogenesis. 2017; 38: 177-83. https://doi.org/10.1093/carcin/bgw204.

11. Li J, Zou L, Zhou Y, Li L, Zhu Y, Yang Y, Gong Y, Lou J, Ke J, Zhang Y, Tian J, Zou D, Peng X, et al. A lowfrequency variant in SMAD7 modulates TGF-beta signaling and confers risk for colorectal cancer in Chinese population. Mol Carcinog. 2017; 56: 1798-807. https://doi.org/10.1002/ mc. 22637 .

12. Margueron R, Reinberg D. The Polycomb complex PRC2 and its mark in life. Nature. 2011; 469: 343-9. https://doi. org/10.1038/nature09784.

13. Di Croce L, Helin K. Transcriptional regulation by Polycomb group proteins. Nat Struct Mol Biol. 2013; 20: 1147-55. https://doi.org/10.1038/nsmb.2669.

14. Kim KH, Roberts CW. Targeting EZH2 in cancer. Nat Med. 2016; 22: 128-34. https://doi.org/10.1038/nm.4036.

15. Varambally S, Dhanasekaran SM, Zhou M, Barrette TR, Kumar-Sinha C, Sanda MG, Ghosh D, Pienta KJ, Sewalt
RG, Otte AP, Rubin MA, Chinnaiyan AM. The polycomb group protein EZH2 is involved in progression of prostate cancer. Nature. 2002; 419: 624-9. https://doi.org/10.1038/ nature 01075.

16. Bracken AP, Pasini D, Capra M, Prosperini E, Colli E, Helin $\mathrm{K}$. EZH2 is downstream of the pRB-E2F pathway, essential for proliferation and amplified in cancer. EMBO J. 2003; 22: 5323-35. https://doi.org/10.1093/emboj/cdg542.

17. Chang WS, Liao $\mathrm{CH}$, Tsai $\mathrm{CW}, \mathrm{Hu} \mathrm{PS}, \mathrm{Wu} \mathrm{HC}$, Hsu SW, Hsiao CL, Hsu CH, Hung YW, Bau DT. Association of Enhancer of Zeste 2 (EZH2) Genotypes with Bladder Cancer Risk in Taiwan. Anticancer Res. 2016; 36: 4509-14. https://doi.org/10.21873/anticanres.10997.

18. Su KJ, Lin CW, Chen MK, Yang SF, Yu YL. Effects of $\mathrm{EZH} 2$ promoter polymorphisms and methylation status on oral squamous cell carcinoma susceptibility and pathology. Am J Cancer Res. 2015; 5: 3475-84.

19. Fornaro L, Faviana P, De Gregorio V, Vivaldi C, Paolicchi E, Masi G, Loupakis F, Sensi E, Lupi C, Fontanini G, Wang Y, Danesi R, Falcone A, et al. Molecular and pathological characterization of the EZH2 rs3757441 single nucleotide polymorphism in colorectal cancer. BMC Cancer. 2015; 15: 874. https://doi.org/10.1186/s12885-015-1889-2.

20. Wang J, Ma ZB, Li K, Guo GH. Association between EZH2 polymorphisms and colorectal cancer risk in Han Chinese population. Med Oncol. 2014; 31: 874. https://doi. org/10.1007/s12032-014-0874-y.

21. Helin K, Dhanak D. Chromatin proteins and modifications as drug targets. Nature. 2013; 502: 480-8. https://doi. org/10.1038/nature12751.

22. Simon JA, Kingston RE. Occupying chromatin: Polycomb mechanisms for getting to genomic targets, stopping transcriptional traffic, and staying put. Mol Cell. 2013; 49: 808-24. https://doi.org/10.1016/j.molcel.2013.02.013.

23. Comet I, Riising EM, Leblanc B, Helin K. Maintaining cell identity: PRC2-mediated regulation of transcription and cancer. Nat Rev Cancer. 2016; 16: 803-10. https://doi. org/10.1038/nrc.2016.83.

24. Sellers WR, Loda M. The EZH2 polycomb transcriptional repressor--a marker or mover of metastatic prostate cancer? Cancer Cell. 2002; 2: 349-50.

25. Cohen AS, Yap DB, Lewis ME, Chijiwa C, Ramos-Arroyo MA, Tkachenko N, Milano V, Fradin M, McKinnon ML, Townsend KN, Xu J, Van Allen MI, Ross CJ, et al. Weaver Syndrome-Associated EZH2 Protein Variants Show Impaired Histone Methyltransferase Function In Vitro. Hum Mutat. 2016; 37: 301-7. https://doi.org/10.1002/ humu. 22946.

26. Sparmann A, van Lohuizen M. Polycomb silencers control cell fate, development and cancer. Nat Rev Cancer. 2006; 6: 846-56. https://doi.org/10.1038/nrc1991. 